\title{
Simultaneous time-series spectroscopy and multi-band photometry of the sdBV PG $1605+072^{\star}$
}

\author{
S. Falter ${ }^{1}$, U. Heber ${ }^{1}$, S. Dreizler ${ }^{2}$, S. L. Schuh ${ }^{2}$, O. Cordes ${ }^{3}$, and H. Edelmann ${ }^{1}$ \\ 1 Dr. Remeis-Sternwarte, Astronomisches Institut der Universität Erlangen-Nürnberg, Sternwartstr. 7, \\ 96049 Bamberg, Germany \\ 2 Institut für Astronomie und Astrophysik, Universität Tübingen, Sand 1, 72076 Tübingen, Germany \\ 3 Sternwarte der Universität Bonn, Auf dem Hügel 71, 53121 Bonn, Germany
}

Received 11 December 2002 / Accepted 15 January 2003

\begin{abstract}
We present time-series spectroscopy and multi-band photometry of the sdBV PG 1605+072 carried out simultaneously at the Calar Alto 2.2-m and 3.5-m telescopes. The periodogram analysis of the radial velocity curves reveals three frequencies at 2.078, 2.756, and $1.985 \mathrm{mHz}$ for $\mathrm{H}_{\beta}$ and at $2.076,2.753$, and $1.978 \mathrm{mHz}$ for $\mathrm{H}_{\gamma}$. The corresponding radial velocity amplitudes are 12.7, 8.0, and $7.9 \mathrm{~km} \mathrm{~s}^{-1}$ for $\mathrm{H}_{\beta}$ and 14.3, 6.5, and $7.2 \mathrm{~km} \mathrm{~s}^{-1}$ for $\mathrm{H}_{\gamma}$. Furthermore, we found five frequencies that are present in all wavelength bands of the BUSCA photometer. The frequencies detected in the radial velocity curves are recovered by the photometric measurements. Moreover, additional frequencies were present in the periodograms which could not be identified in all four bands simultaneously. The comparison of the amplitudes presented here with previous results from radial velocity and photometric observations of PG 1605+072 shows a significant change or even switch in the power of the modes within short time scales, i.e. about one year. No changes in frequency were registered and the phases of the modes show no wavelength dependency within our multi-band photometry.
\end{abstract}

Key words. stars: subdwarfs - stars: oscillations - stars: horizontal branch - stars: individual: PG 1605+072

\section{Introduction}

Subluminous B (sdB) stars dominate the populations of faint blue stars of our own Galaxy and are found in the disk (field $\mathrm{sdBs}$ ) as well as in globular clusters (Moehler et al. 1997). Furthermore, these stars play an important role trying to explain the "UV upturn phenomenon" observed in elliptical galaxies and galaxy bulges (Greggio \& Renzini 1990, 1999). According to observations with the Ultraviolet Imaging Telescope (Brown et al. 1997) and the Hubble Space Telescope (Brown et al. 2000) sdB stars are sufficiently numerous to be responsible for the excessive UV flux.

It is generally accepted that sdB stars can be identified with models of the extended Horizontal Branch (EHB) burning He in their core (Heber 1986; Saffer et al. 1994). The hydrogen envelope surrounding the core of about half a solar mass is very thin $(<2 \%$ by mass) and therefore inert. These EHB stars will continue their evolution directly towards the white dwarf graveyard avoiding the AGB and planetary nebula phases (Dorman et al. 1993).

Send offprint requests to: $\mathrm{S}$. Falter,

e-mail: falter@sternwarte.uni-erlangen.de

* Based on observations obtained at the German-Spanish Astronomical Center (DSAZ), Calar Alto, operated by the MaxPlanck-Institut für Astronomie Heidelberg jointly with the Spanish National Commission for Astronomy.
How sdB stars evolve towards the EHB with effective temperatures of up to $40000 \mathrm{~K}$ remains a puzzle. The star must have lost all but a tiny fraction of the hydrogen envelope at the same time as the He core has attained the minimum mass $\left(\approx 0.5 M_{\odot}\right)$ required for the He flash. This challenges every mass loss mechanism in aspects of timing and effectivity. Recent findings (Maxted et al. 2001; Saffer et al. 2001; Heber et al. 2002a) emphasize the significance of close binary evolution.

Some of the sdB stars were recently found to exhibit rapid multi-periodic light variations $(P \approx 80-600 \mathrm{~s}$ ) of low amplitudes (a few mmag). They form a new class of pulsating stars named after the prototype EC 14026 stars $^{1}$ (Kilkenny et al. 1997). Since then a relatively large number of new sdB pulsators has been discovered. 31 are known today (Charpinet 2001; Piccioni et al. 2000; Silvotti et al. 2000). The observed brightness variations are caused by radial and non-radial, low degree and low order acoustic pulsation modes. The pulsations in these stars are driven by an opacity bump due to $\mathrm{Fe}$ and other metallic species (Charpinet et al. 1997) at a temperature of $\approx 2 \times 10^{5} \mathrm{~K}$ in the sdB envelope.

Stellar pulsations allow a direct insight into the structure of such stars and therefore into the evolutionary history. The frequencies or periods of the pulsation modes probe the chemical stratification and the mass which otherwise are difficult

\footnotetext{
${ }^{1}$ EC 14026 stars are now officially V361 Hya stars.
} 
or even impossible to determine. The power of asteroseismological tools has been demonstrated in the field of pulsating white dwarfs for which stellar parameters like mass, luminosity or thickness of the envelope were derived (e.g. Winget et al. 1991). In the case of variable sdB stars these parameters will constrain the evolutionary history and consequently shed more light on the origin of these stars.

Identification of pulsation modes (characterized by spherical harmonics with the indices $l$ and $m$ ) is a prerequisite for asteroseismology. Brassard et al. (2001) have successfully carried out an asteroseismological analysis for PG 0014+067. For the first time they were able to determine the stellar mass $\left(M_{\star} / M_{\odot}=0.490 \pm 0.019\right)$ as well as the envelope mass $\left(\log \left(M_{\text {env }} / M_{\odot}\right)=-4.31 \pm 0.22\right)$ and both are in excellent agreement with predictions from evolutionary models (Dorman et al. 1993).

Pulsations produce not only photometric variations but also line profile variations that offer an alternative approach towards mode identification. PG $1605+072$ is the ideal target for this application: it has the longest pulsation periods $(\approx 500 \mathrm{~s})$ which enables spectra with reasonable $S / N$ to be obtained within each pulsation period. Moreover, this star has the largest variations of all known sdBVs (0.2 mag in the optical) and by far the richest frequency spectrum (>50 modes, Kilkenny et al. 1999). A recent spectroscopic study revealed this star to be a rather rapid rotator ( $v \sin i=39 \mathrm{~km} \mathrm{~s}^{-1}$, Heber et al. 1999) which will complicate the identification of modes due to non-linear effects on mode splitting.

This work presented here serves as a feasibility study in order to find out whether an asteroseismologic analysis of PG $1605+072$ is possible. For this purpose we have done simultaneously time-series spectroscopy and multi-band photometry (observations and reductions in Sect. 2). The analysis of the results is presented in Sect. 3. Previously, other groups have done radial velocity studies (O'Toole et al. 2000, 2002; Woolf et al. 2002) or photometric campaigns (e.g. Kilkenny et al. 1999) on PG 1605+072. Simultaneous multi-band photometry has not been observed before. This enables us to study the temporal evolution of the frequencies and amplitudes of the pulsation modes (Sect. 4). Finally, we discuss our results and give a brief outlook to future work in Sect. 5.

\section{Observations and data reduction}

\subsection{Time-series spectroscopy}

We obtained time resolved longslit spectra for the sdBV PG 1605+072 during one observing run. The observations were carried out with the 3.5-m telescope at the DSAZ, Calar Alto, Spain and the TWIN spectrograph (two SITe-CCDs with $2048 \times 800$ pixels à $15 \mu \mathrm{m}$ ). The time allocation committee awarded two nights on May 14 and 15, 2001. Because of rain during the first night data were only obtained during the second night ( $5 \mathrm{~h} 27 \mathrm{~m}$ of data). The passage of clouds and variable seeing affected the quality of the data considerably. In order to achieve an adequate time resolution observations were carried out in trailing mode which means that the telescope tracking is tuned such that the star moves slowly along the slit in N-S direction. The drift velocity was chosen at $270^{\prime \prime}$ per hour so that a time resolution of $15 \mathrm{~s}$ per pixel resulted. The time resolution is dependent on the seeing conditions and thus varied during the night. The slit width was $1.5^{\prime \prime}$. We chose the gratings T05 and T06 (36 $\mathrm{A} / \mathrm{mm})$ corresponding to a spectral resolution of $\approx 1 \AA$. The spectral ranges from $3860-4960 \AA$ in the blue and from 5880-6920 $\AA$ in the red were covered. Moreover, to decrease the readout time and the noise level the data were binned $(1 \times 2)$.

\subsection{Multi-band photometry}

Simultaneously with the time resolved spectroscopy of the target star its light curve was measured in four wavelength bands at the 2.2-m telescope at Calar Alto. At this telescope five nights between May 14 and 18, 2001 were awarded. No data could be taken during the first night and from the middle of the fourth night until the end of the run due to bad weather conditions. $12 \mathrm{~h} 27 \mathrm{~m}$ of pure observation time resulted in 880 measurements. The telescope was equipped with the new multi-band photometer BUSCA (Reif et al. 1999; Cordes et al. in prep.) which is able to measure in four photometric bands simultaneously. Three beamsplitters split the incoming light into four beams that feed four $4 \mathrm{k} \times 4 \mathrm{k}$ CCDs. Only Strømgren filters were available for BUSCA. Use of these filters would have further reduced the $S / N$. Therefore, no filters were inserted and the beamsplitters served as non-overlapping broad band filters. The transmission curves of the four bands are shown in Fig. 1. These curves convoluted with the quantum efficiency curves of the CCDs provide the pass bands for our observations. The four wavelength bands are denoted by " $U V_{\mathrm{B}}$ ", " $B_{\mathrm{B}}$ ", " $R_{\mathrm{B}}$ " and " $N I R_{\mathrm{B}}$ " throughout the paper following the notation of Cordes et al. (in prep.). We chose an exposure time of $15 \mathrm{~s}$ which provides a good $S / N$ and a reasonable coverage of the main frequencies. $2 \times 2$ binning (resolution: $0.17^{\prime \prime}$ per unbinned pixel) and the windowing option (reading out $346 \times 400$ pixels of the CCDs) helped to reduce the full cycle time to $51 \mathrm{~s}$.

The brightness variations of PG $1605+072$ were detected by relative photometry. For this purpose two stars of similar brightnesses in the $R$ band $\left(R_{1}=13.2\right.$ and $\left.R_{2}=13.4\right)$ next to our target star were used.

\subsection{Data reduction}

Data reduction was done using the IDL based software SPEX (Long slit SPectrum EXtraction package ${ }^{2}$ ), and TRIPP (Time Resolved Imaging Photometry Package, see Schuh et al. 1999). TRIPP is based on the CCD photometry routines written by R. D. Geckeler (1998) and performs aperture photometry. The most important step is the determination of the relative flux of the target star with one or more stars as comparison objects. The detection of variations of the order of a few mmag is only possible when comparison stars and sky background are

\footnotetext{
2 see http://astro.uni-tuebingen.de/ schuh/spex/ index.html
} 


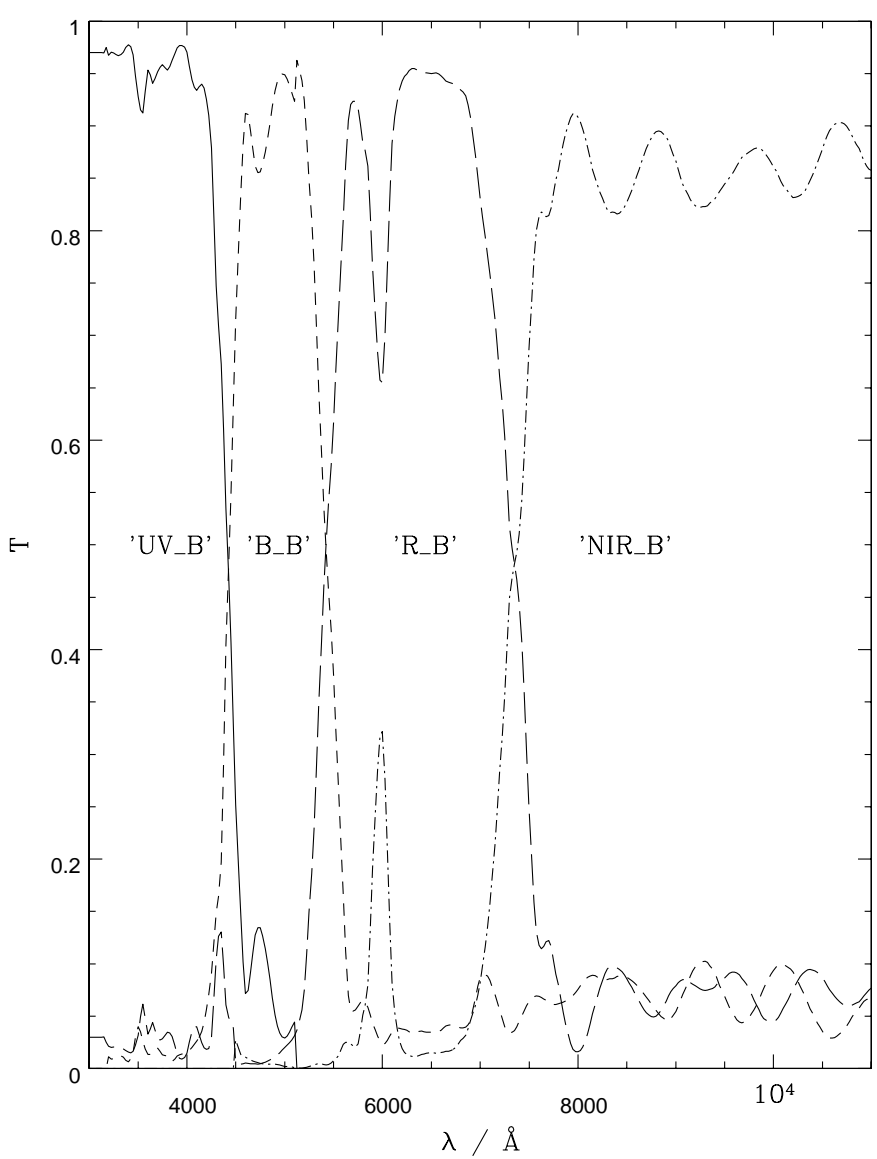

Fig. 1. Transmission curves of the four BUSCA wavelength bands. The four ranges are denoted by " $U V_{\mathrm{B}}$ " (central wavelength: $3600 \AA$ ),

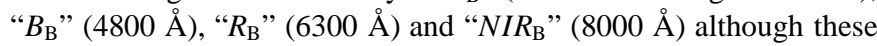
are not comparable to the classical Johnson UBVRI system.

recorded simultaneously (especially under comparatively poor conditions).

SPEX is designed for the reduction of long slit spectra. The reduction comprises of the standard procedures flatfielding, biasing, cosmic ray event extraction, and 2-D wavelength calibration. The latter is very important because we extract spectra from single CCD rows in order to establish the time-series. Therefore, we have to ensure that the position of the comparison lines perpendicular to the dispersion direction doesn't change. In fact, these positions vary and thus we correct for that by fitting a polynomial. After the calibration of one CCD row the resulting dispersion relation is applied to every single CCD row.

One crucial point was the calibration of the time axis. As a consequence of the trailing mode observation the seeing disk moves slowly across the CCD and therefore the signal smears over the trailing direction. The initial point of time of the measurement and the cycle time were determined by fitting Gaussian profiles to the rising flank of the star's signal. Finally, radial velocities were derived by fitting Lorentz profiles to the Balmer lines $\mathrm{H}_{\alpha}-\mathrm{H}_{8}$ as well as to $\mathrm{He}_{\mathrm{I}} 4471 \AA$ and $\mathrm{He}_{\text {II }} 4686 \AA$.

\section{Analysis of the time-series}

Both the spectroscopic as well as the photometric time-series were analysed with the program package TRIPP. It enables the calculation of periodograms, confidence levels and fits with multiple sine functions (see Dreizler et al. 2002 for more details).

\subsection{Spectroscopy}

We were able to derive accurate radial velocity curves for the Balmer lines $\mathrm{H}_{\beta}$ and $\mathrm{H}_{\gamma}$ (see Fig. 2). The frequency resolution for this run is calculated to be $51 \mu \mathrm{Hz}$. Trailing mode observations require stable weather conditions so that the intensity of the star's signal is almost constant during one exposure. Thus bad seeing and transparency changes due to the passage of small clouds influence the quality of the data rather strongly. Because of these disturbances the quality of the radial velocity curves varies with time. The radial velocity curves of the other Balmer lines and the He lines turned out to be too noisy for a quantitative analysis and are not further discussed. Better conditions should allow a more extensive study of radial velocity changes in this star.

The Lomb-Scargle periodograms calculated from the radial velocity curves (see Fig. 3) show peaks clearly surpassing the $3 \sigma$ confidence level. Applying a prewhitening procedure to the data three frequencies and their amplitudes (see Table 1) were found for both Balmer lines investigated. Other peaks that seem to lie above the $3 \sigma$ level could not be extracted by our procedure. The dominant frequency at $2.076 \mathrm{mHz}$ has the largest velocity amplitude with $12.7 \mathrm{~km} \mathrm{~s}^{-1}$ for $\mathrm{H}_{\beta}$ and $14.3 \mathrm{~km} \mathrm{~s}^{-1}$ for $\mathrm{H}_{\gamma}$. The amplitudes of the other two frequencies are lower than the strongest one with values ranging from 6 to $8 \mathrm{~km} \mathrm{~s}^{-1}$. We determined the amplitude accuracy $\Delta A$ by calculating the median value of the white noise in the frequency range $3-7 \mathrm{mHz}$ where almost no power arises in the frequency spectrum. According to this, we found $\Delta A=1.5 \mathrm{~km} \mathrm{~s}^{-1}$ for $\mathrm{H}_{\beta}$ and $\Delta A=1.0 \mathrm{~km} \mathrm{~s}^{-1}$ for $\mathrm{H}_{\gamma}$.

\subsection{Multi-band photometry}

BUSCA is a unique instrument which enables the measurement in four different wave bands simultaneously. As a result we obtained four light curves.

Figure 4 shows the Lomb-Scargle periodograms of all BUSCA bands. They are quite similar to the periodograms derived from the radial velocity curves (Fig. 3). This data set spans over three nights so that daily aliases are clearly visible and the frequency resolution is much better at $\Delta v=5.68 \mu \mathrm{Hz}$ (compared to $51 \mu \mathrm{Hz}$ for the spectroscopy). Again we applied the prewhitening technique in order to remove all significant peaks from the periodogram and to obtain the amplitudes for each frequency. As before, the horizontal line in the diagrams represents the $3 \sigma$ confidence level above which we assume the detected frequencies to be real. In all four wave bands five peaks with the same frequencies can be identified (see Tables 2 and 3). The dominant frequency is again found at $2.076 \mathrm{mHz}$ and therefore confirms the results from 

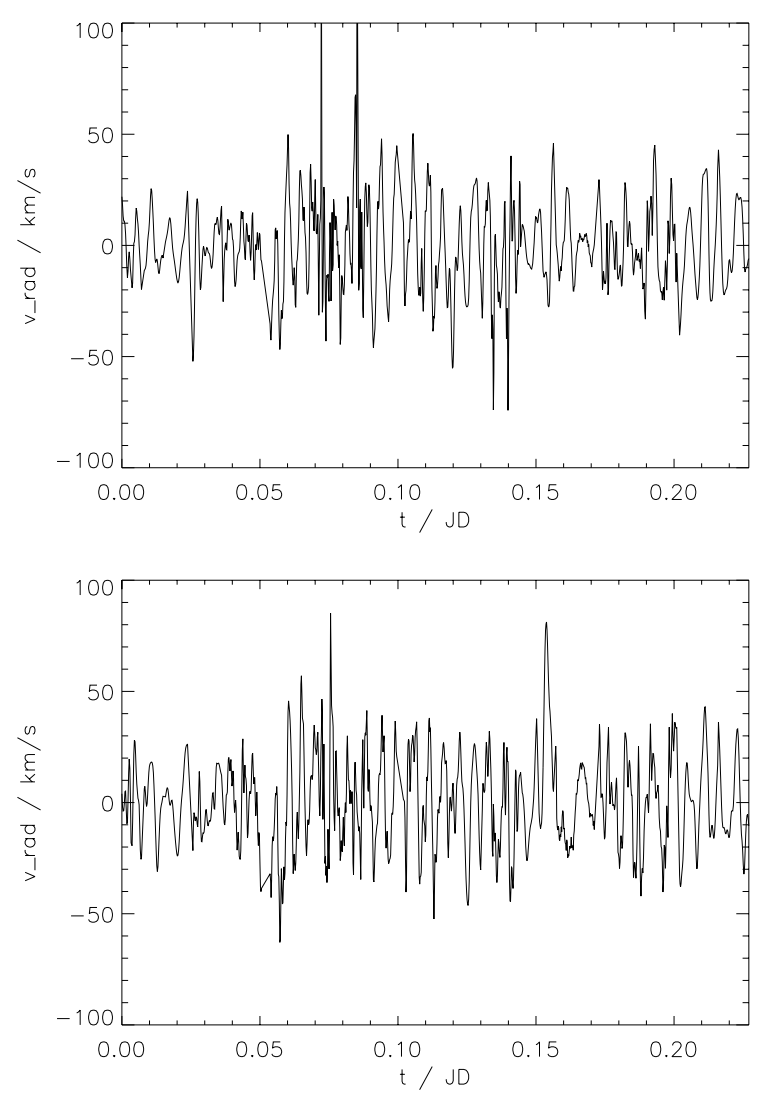

Fig. 2. Radial velocity curve for $\mathrm{H}_{\beta}$ (upper panel) and $\mathrm{H}_{\gamma}$ (lower panel); artefacts due to poor weather conditions were not removed.

spectroscopy. Furthermore, additional frequencies were found in the region around $2.74-2.78 \mathrm{mHz}$ but these peaks are closely spaced so that a corresponding identification in all BUSCA bands due to the medium frequency resolution was not possible. Peaks that fall below the $3 \sigma$ confidence level were not removed from the periodograms.

The amplitudes of the brightness variations are measured in fractional intensity. The light curves are normalized to the fraction of intensity $\delta I / I$ and the amplitudes were then converted to mmag. This unit will be used throughout the whole paper. The accuracy of these amplitudes are calculated in the same way as we did it for the radial velocity amplitudes. Here we used the median level of the white noise in the range 3-5 $\mathrm{mHz}$. The accuracies for the BUSCA wavebands are $1.52 \mathrm{mmag}$ for " $U V_{\mathrm{B}}$ ", $1.53 \mathrm{mmag}$ for " $B_{\mathrm{B}}$ ", $1.12 \mathrm{mmag}$ for " $R_{\mathrm{B}}$ " and $1.37 \mathrm{mmag}$ for "NIR $\mathrm{B}$ ", respectively.

Figure 5 shows the semi amplitudes of four selected frequencies as a function of effective wavelength of the bands. In Fig. 6 we display the relative change of the semi amplitude of each waveband. The deviation with respect to the mean is largest for the " $U V_{\mathrm{B}}$ " band. The other channels, considered separately, behave rather similar showing much smaller deviations from the mean brightness. This is explained through the fact that the " $U V_{\mathrm{B}}$ " band lies blueward to the Balmer jump and the other redward of it. The opacity changes a lot across this wavelength range and thus the stellar flux originates from different atmospheric depths.
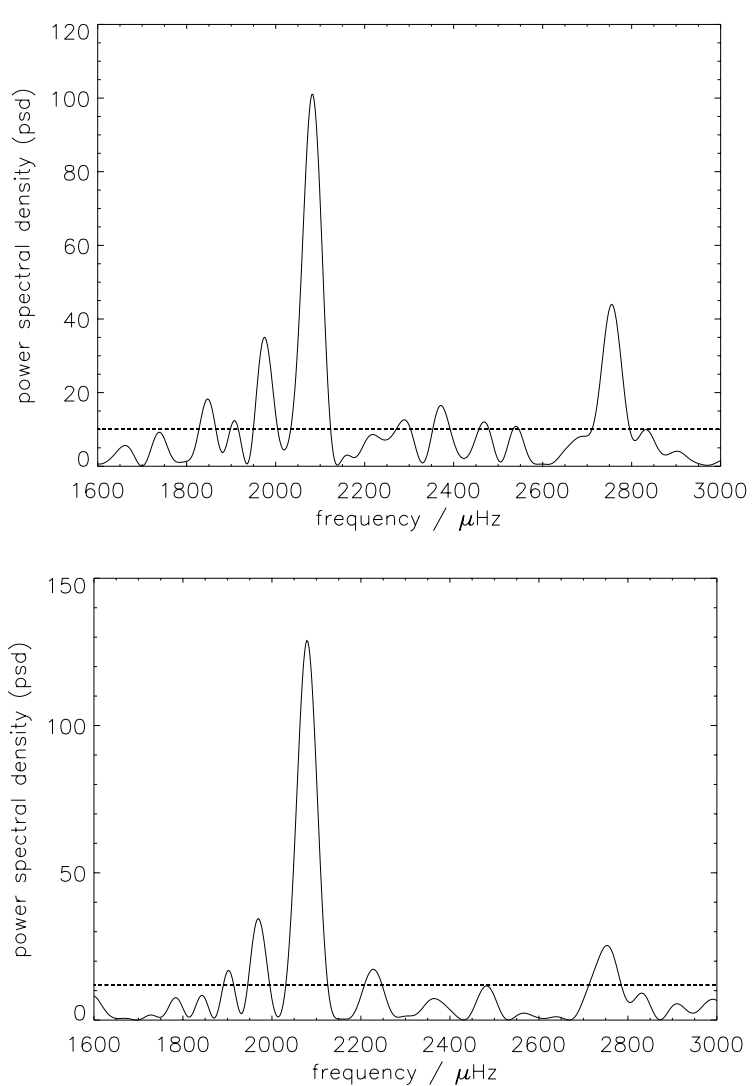

Fig. 3. Lomb-Scargle periodogram of the radial velocity curve of $\mathrm{H}_{\beta}$ (upper panel) and $\mathrm{H}_{\gamma}$ (lower panel). The power spectral density (psd) is a measure for the probability that a period is present in the radial velocity curve or light curve. The horizontal line represents the confidence level of $99 \%$ ( $3 \sigma$-level).

Furthermore, we used the phases which are delivered by the sine fit procedure (see Tables 2 and 3 ) in order to test whether there is any wavelength dependency. The phase values are normalized to unity. Figure 7 shows the deviations of the phases with respect to the mean value of all four wavebands for the four selected frequencies of Fig. 5. The error bars are calculated from

$A=\operatorname{Re}\{A\} \sin \phi$.

The phase error $\Delta \phi$ can be calculated from the amplitude uncertainty $\Delta A$ according to

$\Delta \phi=\arctan \left(\frac{\Delta A}{A}\right)$.

The two strongest frequencies at 2.076 and $1.986 \mathrm{mHz}$ show no wavelength dependent behavior within the error margins. The deviations for the other two frequencies from the mean value are slightly larger than the formal errors. However, we regard this as insignificant, and conclude that we cannot find any wavelength dependency of the phases of the modes.

\section{Comparison with previous investigations}

Other groups have carried out similar studies already, either time-series spectroscopy (O'Toole et al. 2000; O'Toole et al. 2002; Woolf et al. 2002) or photometry (Kilkenny et al. 1999). 

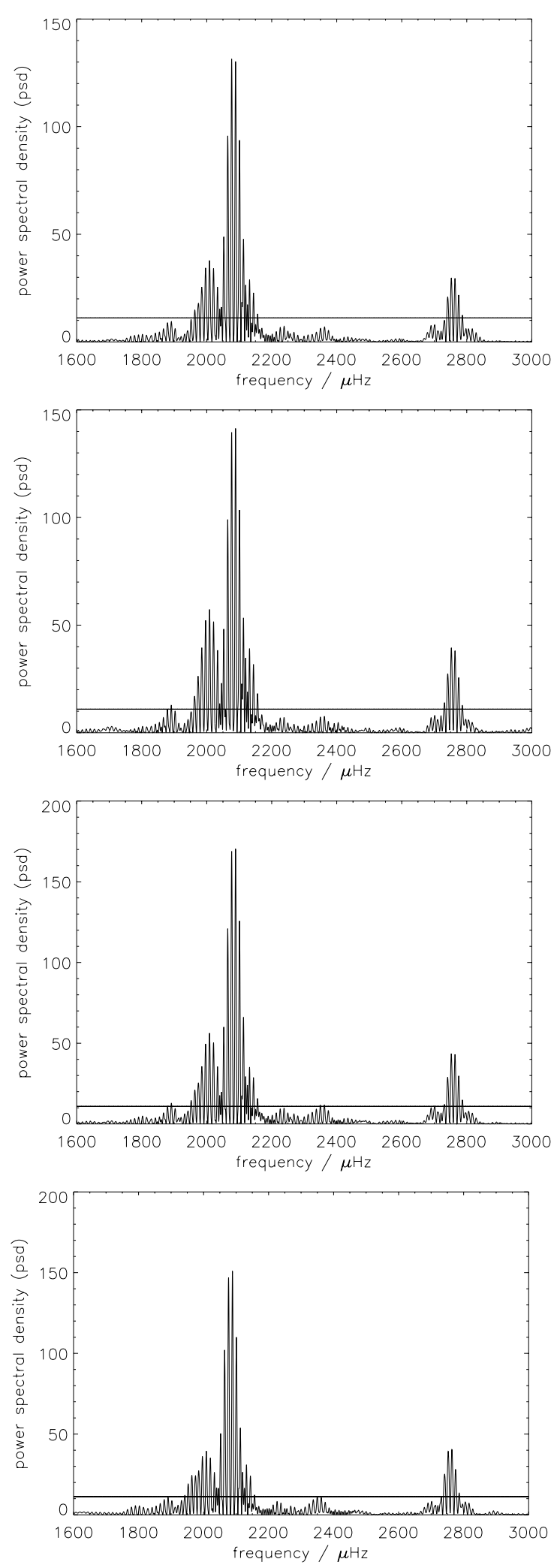

Fig. 4. Lomb-Scargle periodogram of the light curves of the " $U V_{\mathrm{B}}$ ", " $B_{\mathrm{B}}$ ", " $R_{\mathrm{B}}$ " and "NIR $R_{\mathrm{B}}$ " band (from top to bottom) of the BUSCA camera. The horizontal line in each panel represents the confidence level of $99 \%(3 \sigma$-level).

O'Toole et al. (2000) have collected monochromatic photometric data, too, but they are not strictly simultaneous. We shall compare our results with their results in this section.

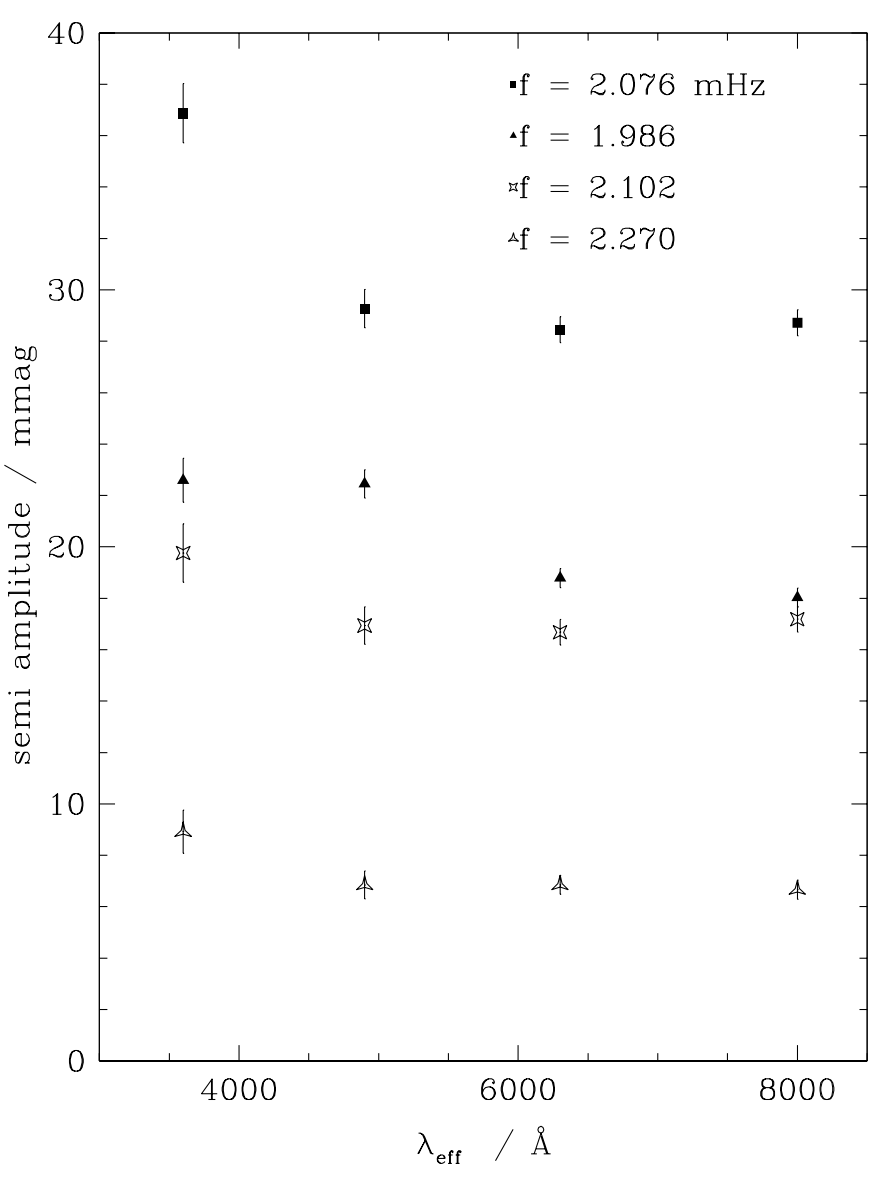

Fig. 5. Semi amplitudes of four frequencies as a function of effective wavelength. The error bars are $1 \sigma$ errors and are calculated by means of a $\chi^{2}$ method.

The photometric multi-site campaign of Kilkenny et al. (1999) discovered more than 50 frequencies in their frequency analysis of PG 1605+072. The five frequencies $(2.076,1.9865$, 2.102, 2.2695 and $1.8912 \mathrm{mHz}$ ) detected in all BUSCA bands of our data were already present in their data. Some of the others which we found additionally (e.g. $4.0631 \mathrm{mHz}$ ) can be identified with frequencies from Kilkenny et al. (1999) and others can not (in particular at $2.7866 \mathrm{mHz}$ ). A closer inspection of these frequencies shows that all of them are very close to one-day aliases $( \pm 11.57 \mu \mathrm{Hz})$ of the extracted frequencies of our or of Kilkenny's data to within the frequency resolution of $5.68 \mu \mathrm{Hz}$. Small deviations of our measurements from those of Kilkenny et al. (1999) can be explained by the shorter time spanned by our data $(\approx 48 \mathrm{~h}$ compared to $\approx 217.6 \mathrm{~h}$, frequency resolution: $5.68 \mu \mathrm{Hz}$ compared to $1.28 \mu \mathrm{Hz}$ ). In contrast to our analysis, Kilkenny et al. (1999) were able to resolve closely spaced frequencies, e.g. at $2.1017 \mu \mathrm{Hz}$ and $2.1033 \mu \mathrm{Hz}$ (see Table 2). Since 1997 the relative power within one blend of frequencies may have changed so that the frequency of unresolved peaks in the periodogram changes slightly because the contribution of the weaker feature at that time may have grown in the meantime. We conclude that the frequencies measured in the BUSCA light curves are consistent with those of Kilkenny et al. (1999). The frequencies are stable in time to within our measurement errors. 
Table 1. Radial velocities $(R V)$ of PG $1605+072$ derived for $\mathrm{H}_{\beta}$ and $\mathrm{H}_{\gamma}$ and for comparison of the other radial velocity studies. Woolf et al. (2002) measured their radial velocities from shifts of the whole spectrum. The radial velocities of O'Toole et al. (2002) are measured for all Balmer lines (velocity error: $\approx 0.4 \mathrm{~km} \mathrm{~s}^{-1}$ ).

\begin{tabular}{cccccccccc}
\hline \hline & $\mathrm{H}_{\beta}$ & & & $\mathrm{H}_{\gamma}$ & & Woolf et al. (2002) & \multicolumn{3}{c}{ O'Toole et al. (2002) } \\
\hline $\begin{array}{c}f \\
{[\mathrm{mHz}]}\end{array}$ & $\begin{array}{c}P \\
{[\mathrm{~s}]}\end{array}$ & $\begin{array}{c}R V \\
{\left[\mathrm{~km} \mathrm{~s}^{-1}\right]}\end{array}$ & $\begin{array}{c}f \\
{[\mathrm{mHz}]}\end{array}$ & $\begin{array}{c}P \\
{[\mathrm{~s}]}\end{array}$ & $\begin{array}{c}R V \\
{\left[\mathrm{~km} \mathrm{~s}^{-1}\right]}\end{array}$ & $\begin{array}{c}P \\
{[\mathrm{~s}]}\end{array}$ & $\begin{array}{c}R V \\
{\left[\mathrm{~km} \mathrm{~s}^{-1}\right]}\end{array}$ & $\begin{array}{c}P \\
{[\mathrm{~s}]}\end{array}$ & $\begin{array}{c}R V \\
{\left[\mathrm{~km} \mathrm{~s}^{-1}\right]}\end{array}$ \\
\hline 2.078 & 481.28 & 12.7 & 2.076 & 481.66 & 14.3 & $481.7 / 475.3$ & $3.9 / 4.0$ & $480 / 475$ & $4.3 / 8.5$ \\
2.756 & 362.89 & 8.0 & 2.753 & 363.21 & 6.5 & $363.2 / 366.2$ & $3.0 / 6.1$ & 365 & 7.2 \\
1.985 & 503.79 & 7.9 & 1.978 & 505.77 & 7.2 & 502.0 & 3.9 & 504 & 4.1 \\
& & & & & & 527.1 & 2.7 & & \\
\hline
\end{tabular}

Table 2. Frequencies and corresponding periods as well as amplitudes for PG $1605+072$ in the first two BUSCA wavebands (" $U V_{\mathrm{B}}$ ” and " $B_{\mathrm{B}}$ ”). The errors of the periods and amplitudes are the formal fit errors from the sine fit procedure. The left column shows the corresponding values derived in Kilkenny et al. (1999) as a comparison to the values found in this work. The two frequencies marked with a star can not be resolved in our data but can in the data of Kilkenny (see Sect. 4 for the discussion). The phases derived within the sine fit procedure are denoted by $\phi$ and are discussed in Sect. 3 as well as the determination of the phase errors.

\begin{tabular}{|c|c|c|c|c|c|c|c|c|c|}
\hline Kilk.'99 & & & & $" U V_{\mathrm{B}} "$ & & & $" B_{\mathrm{B}} "$ & & \\
\hline$f_{\text {Kilk99 }}$ & $P_{\text {Kilk99 }}$ & $A_{\text {Kilk99 }}$ & $f$ & $P$ & $A$ & $\phi$ & $P$ & $A$ & $\phi$ \\
\hline$[\mathrm{mHz}]$ & {$[\mathrm{s}]$} & [mmag] & {$[\mathrm{mHz}]$} & {$[\mathrm{s}]$} & [mmag] & & {$[\mathrm{s}]$} & [mmag] & \\
\hline 2.0758 & 481.75 & 27.4 & 2.0760 & $481.69 \pm 0.02$ & $36.88 \pm 0.12$ & $0.684 \pm 0.006$ & $481.69 \pm 0.02$ & $29.27 \pm 0.07$ & $0.700 \pm 0.008$ \\
\hline 1.9853 & 503.70 & 3.3 & 1.9861 & $503.51 \pm 0.03$ & $22.59 \pm 0.09$ & $0.953 \pm 0.010$ & $503.60 \pm 0.02$ & $22.45 \pm 0.06$ & $0.951 \pm 0.010$ \\
\hline$\star 2.1017$ & 475.82 & 15.4 & 2.1020 & $475.74 \pm 0.04$ & $19.76 \pm 0.11$ & $0.026 \pm 0.011$ & $475.82 \pm 0.03$ & $16.94 \pm 0.07$ & $0.000 \pm 0.013$ \\
\hline$\star 2.1033$ & 475.45 & 15.9 & & & & & & & \\
\hline 2.7613 & 362.15 & 1.8 & 2.7631 & $361.92 \pm 0.03$ & $16.01 \pm 0.16$ & $0.051 \pm 0.014$ & $361.97 \pm 0.03$ & $10.54 \pm 0.07$ & $0.043 \pm 0.021$ \\
\hline 2.7663 & 361.49 & 2.0 & 2.7668 & $361.43 \pm 0.06$ & $10.94 \pm 0.15$ & $0.192 \pm 0.020$ & & & \\
\hline- & - & - & 2.7554 & & & & $363.10 \pm 0.02$ & $14.97 \pm 0.07$ & $0.080 \pm 0.015$ \\
\hline 2.2701 & 440.51 & 5.2 & 2.2700 & $440.52 \pm 0.05$ & $8.92 \pm 0.08$ & $0.144 \pm 0.025$ & $440.54 \pm 0.04$ & $6.85 \pm 0.05$ & $0.148 \pm 0.032$ \\
\hline 1.8914 & 528.70 & 13.9 & 1.8915 & $528.69 \pm 0.09$ & $7.65 \pm 0.09$ & $0.675 \pm 0.029$ & $528.79 \pm 0.05$ & $8.25 \pm 0.05$ & $0.643 \pm 0.027$ \\
\hline 2.7173 & 368.01 & 0.6 & 2.7191 & $367.77 \pm 0.06$ & $7.45 \pm 0.10$ & $0.897 \pm 0.029$ & & & \\
\hline 2.3920 & 418.05 & 2.2 & 2.3921 & & & & $418.05 \pm 0.04$ & $7.87 \pm 0.05$ & $0.833 \pm 0.028$ \\
\hline- & - & - & 4.0748 & & & & $245.41 \pm 0.02$ & $4.39 \pm 0.05$ & $0.549 \pm 0.049$ \\
\hline
\end{tabular}

Table 3. Frequencies and corresponding periods as well as amplitudes for PG $1605+072$ in the last two BUSCA wavebands (" $R_{\mathrm{B}}$ ” and " $N I R_{\mathrm{B}}$ ”). The errors of the periods and amplitudes are the formal fit errors from the sine fit procedure. The left column shows the corresponding values derived in Kilkenny et al. (1999) as a comparison to the values found in this work. The phases derived within the sine fit procedure are denoted by $\phi$ and are discussed in Sect. 3 as well as the determination of the phase errors.

\begin{tabular}{|c|c|c|c|c|c|c|c|c|c|}
\hline Kilk.'99 & & & & $" R_{\mathrm{B}} "$ & & & $" N I R_{\mathrm{B}} "$ & & \\
\hline $\begin{array}{c}f_{\text {Kilk99 }} \\
{[\mathrm{mHz}]}\end{array}$ & $\begin{array}{c}P_{\text {Kilk99 }} \\
{[\mathrm{s}]}\end{array}$ & $\begin{array}{c}A_{\text {Kilk99 }} \\
{[\mathrm{mmag}]}\end{array}$ & $\begin{array}{c}f \\
{[\mathrm{mHz}]}\end{array}$ & $\begin{array}{c}P \\
{[\mathrm{~s}]}\end{array}$ & $\begin{array}{c}A \\
{[\mathrm{mmag}]}\end{array}$ & $\phi$ & $\begin{array}{c}P \\
{[\mathrm{~s}]}\end{array}$ & $\begin{array}{c}A \\
{[\mathrm{mmag}]}\end{array}$ & $\phi$ \\
\hline 2.0758 & 481.75 & 27.4 & 2.0759 & $481.71 \pm 0.01$ & $28.45 \pm 0.05$ & $0.691 \pm 0.006$ & $481.73 \pm 0.02$ & $28.72 \pm 0.07$ & $0.684 \pm 0.007$ \\
\hline 1.9853 & 503.70 & 3.3 & 1.9858 & $503.58 \pm 0.01$ & $18.79 \pm 0.04$ & $0.948 \pm 0.009$ & $503.61 \pm 0.02$ & $18.02 \pm 0.05$ & $0.936 \pm 0.011$ \\
\hline 2.1017 & 475.82 & 15.4 & 2.1020 & $475.76 \pm 0.02$ & $16.68 \pm 0.05$ & $0.028 \pm 0.010$ & $475.70 \pm 0.03$ & $17.18 \pm 0.07$ & $0.057 \pm 0.012$ \\
\hline- & - & - & 2.7530 & $363.23 \pm 0.01$ & $17.15 \pm 0.04$ & $0.054 \pm 0.010$ & & & \\
\hline - & - & - & 2.7866 & $358.87 \pm 0.03$ & $6.22 \pm 0.04$ & $0.213 \pm 0.026$ & & & \\
\hline 2.7427 & 364.60 & 15.1 & 2.7427 & & & & $364.60 \pm 0.02$ & $10.37 \pm 0.06$ & $0.043 \pm 0.019$ \\
\hline - & - & - & 2.7637 & & & & $361.84 \pm 0.02$ & $13.40 \pm 0.06$ & $0.057 \pm 0.015$ \\
\hline 2.2701 & 440.51 & 5.2 & 2.2700 & $440.56 \pm 0.03$ & $6.85 \pm 0.04$ & $0.133 \pm 0.024$ & $440.60 \pm 0.04$ & $6.66 \pm 0.05$ & $0.183 \pm 0.030$ \\
\hline 1.8914 & 528.70 & 13.9 & 1.8914 & $528.71 \pm 0.04$ & $6.79 \pm 0.04$ & $0.670 \pm 0.024$ & $528.90 \pm 0.06$ & $6.88 \pm 0.05$ & $0.625 \pm 0.029$ \\
\hline 2.3920 & 418.05 & 2.2 & 2.3920 & $418.06 \pm 0.03$ & $5.80 \pm 0.04$ & $0.811 \pm 0.028$ & $417.94 \pm 0.04$ & $6.44 \pm 0.05$ & $0.821 \pm 0.031$ \\
\hline 4.0618 & 246.19 & 1.2 & 4.0624 & $246.16 \pm 0.01$ & $4.47 \pm 0.04$ & $0.458 \pm 0.036$ & $246.17 \pm 0.02$ & $5.38 \pm 0.05$ & $0.413 \pm 0.037$ \\
\hline
\end{tabular}




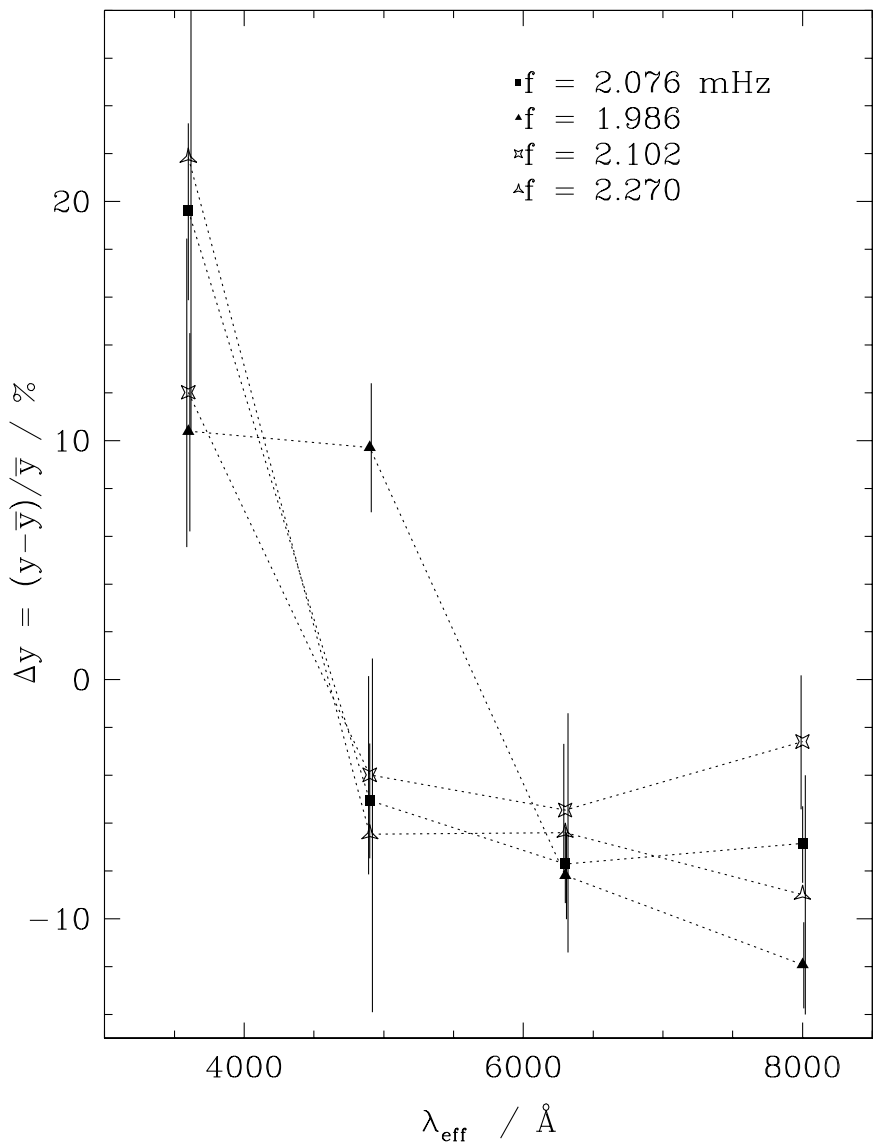

Fig. 6. Relative semi amplitudes of the four frequencies from Fig. 5. These are calculated by $\Delta y=(y-\bar{y}) / \bar{y}$. $y$ is the semi amplitude of the wavebands. The error bars are the formal $1 \sigma$ errors of the sine fit procedure.

Also the frequencies discovered in the radial velocity curves are consistent with both the BUSCA and the Kilkenny values. The time basis of the spectroscopic data is too short in comparison to the long-term photometry to resolve the frequencies. This is also true for most of the other spectroscopic feasibility studies mentioned before. Only O'Toole et al. (2002) had a sufficiently long baseline of $\approx 70 \mathrm{~d}$. Thus, we discuss only the relative distribution of the power of the pulsation frequencies. As can be seen in Fig. 3 the main power is located around $2.076 \mathrm{mHz}$. Significant power arises in the frequency range $2.74-2.78 \mathrm{mHz}$ but it falls off compared to the former. The same distribution of pulsation power was detected by the multi-site photometric campaign of Kilkenny et al. (1999) who carried out their observations in April and May 1997. Two years later, in July and August 1999, O'Toole et al. (2000) recovered the same frequency pattern but discovered that the amplitudes had changed and, in particular, that no power was detected at $2.076 \mathrm{mHz}$. Another year later (in May 2000) O'Toole et al. (2002) and Woolf et al. (2002) gathered data again. Both groups discovered a drastic change in the relative power distribution: The power at $2.076 \mathrm{mHz}$ appeared again and was at that time weaker than that in the frequency range $2.74-2.78 \mathrm{mHz}$. This means that the power switches within a few years. Our data which were taken in May 2001, i.e. another year later,

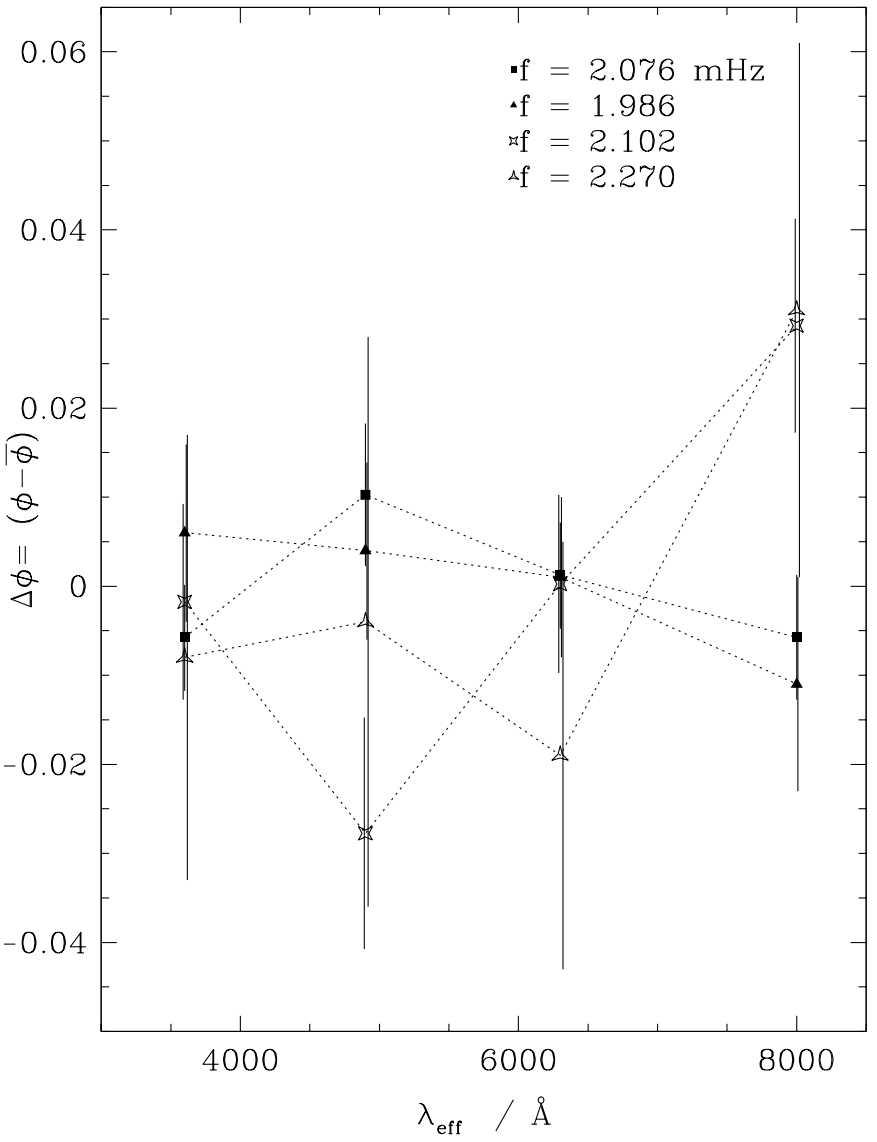

Fig. 7. Deviation of the phases from the mean value calculated from the four frequency bands for the frequencies given in Fig. 5. The error bars are calculated from the amplitude errors as described in the text.

show that the power distribution switched back to that of 1997 measured by Kilkenny et al. (1999).

\section{Conclusion}

Our spectroscopic feasibility study based on data taken in May 2001 confirms earlier studies from observations in July/August 1999 and May 2000. All these investigations have shown that it is possible to measure accurate radial velocity curves for PG 1605+072. Three to five frequencies could be detected in the periodograms. The detected frequencies found in the radial velocity studies are very stable (see Tables 2 and 3). However, the amplitudes of the frequencies show drastic temporal changes over a few years. We also have simultaneously measured photometric variations of PG 1605+072 in four wavebands for the first time. The amplitudes in the " $U V_{\mathrm{B}}$ " band are found to be significantly larger than at larger wavelengths (" $B_{\mathrm{B}}$ ", " $R_{\mathrm{B}}$ " and "NIR $R_{\mathrm{B}}$ "). No phase shifts between the four light curves can be detected.

The time basis of all observations is much too short to resolve the full frequency spectrum of this star. More than 50 frequencies have been resolved in the long photometric study of Kilkenny et al. (1999). Consequently, a new data set with a longer time basis is needed. For that reason we organised a combined photometric and spectroscopic multi-site campaign (PI: U. Heber), termed the MSST (Multi Site Spectroscopic 
Telescope) project (Heber et al. 2002b). These observations covered a period of one month in May/June 2002. This project is unique in that it combines photometric and spectroscopic data. The data still have to be reduced and analysed. A lot of work remains to be done. Hopefully, we will be able to identify pulsation modes within this large data set and carry out a detailed asteroseismological investigation of PG 1605+072. The feasibility study presented in this paper in concert with all other equivalent studies mentioned in Sect. 4 have paved the way for this aim.

Acknowledgements. The authors would like to thank the Calar Alto staff for their support during our observation run in May 2001. Financial support by the Deutsches Zentrum für Luft- und Raumfahrt (DLR) under grant 50 OR 96029-ZA is gratefully acknowledged. We also thank the Deutsche Forschungs Gemeinschaft (DFG) for a travel grant (HE 1356/33-1) to Calar Alto observatory. BUSCA was realised with financial support by the german Bundesministerium für Bildung, Wissenschaft, Forschung und Technologie (BMBF) through grant 03BN114(4) of Verbundforschung Astronomie/Astrophysik. We thank Simon O'Toole for his valuable comments on the text and contributing a routine to calculate the errors of the amplitudes and phases.

\section{References}

Brassard, P., Fontaine, G., Billères, M., et al. 2001, ApJ, 563, 1013

Brown, T. M., Ferguson, H. C., Davidsen, A. F., \& Dorman, B. 1997, ApJ, 482, 685

Brown, T. M., Bowers, C. W., Kimble, R. A., Sweigart, A. V., \& Ferguson, H. C. 2000, ApJ, 532, 308

Charpinet, S. 2001, AN, 322, 387

Charpinet, S., Fontaine, G., Brassard, P., \& Dorman, B. 1996, ApJ, 471, L103

Charpinet, S., Fontaine, G., Brassard, P., Chayer, P., \& Rogers, F. J. 1997, ApJ, 483, L123

Dorman, B., Rood, R. T., \& O’Connell, R. W. 1993, ApJ, 419, 596

Dreizler, S., Schuh, S. L., Deetjen, J. L., Edelmann, H., \& Heber, U. 2002, A\&A, 386, 249

Geckeler, R. D., Ph.D. Thesis, Eberhard-Karls-Universität Tübingen

Greggio, L., \& Renzini, A. 1990, ApJ, 364, 35

Greggio, L., \& Renzini, A. 1999, Mem. S.A.I., 70, 691
Heber, U. 1986, A\&A, 155, 33

Heber, U., Reid, I. N., \& Werner, K. 1999, A\&A, 348, L25

Heber, U., Reid, I. N., \& Werner, K. 2000, A\&A, 363, 198

Heber, U., Moehler, S., Napiwotzki, R., et al. 2002a, A\&A, 383, 938

Heber, U., Dreizler, S., Schuh, S. L., et al. 2002b, in White Dwarfs, ed. D. de Martino, R. Silvotti, J.-E. Solheim, \& R. Kalytis (Dordrecht: Kluwer), in press [astro-ph/0212447]

Kilkenny, D., Koen, C., O’Donoghue, D., \& Stobie, R. S. 1997, MNRAS, 285, 640

Kilkenny, D., Koen, C., O’Donoghue, D., et al. 1999, MNRAS, 303, 525

Maxted, P. F. L., Heber, U., Marsh, T., \& North, R. C. 2001, MNRAS, 326, 1391

Moehler, S., Richtler, T., de Boer, K. S., Dettmar, R. J., \& Heber, U. 1990, A\&AS, 86, 53

Moehler, S., Heber, U., \& Rupprecht, G. 1997, A\&A, 319, 109

O’ Toole, S. J., Bedding, T. R., Kjeldsen, H., et al. 2000, ApJ, 537, L53

O’Toole, S. J., Bedding, T. R., Kjeldsen, H., Dall, T. H., \& Stello, D. 2002, MNRAS, 334, 471

Piccioni, A., Bartolini, C., Bernabei, S., et al. 2000, A\&A, 354, L13

Press, W. H., \& Rybicki, G. B. 1989, ApJ, 338, 277

Reif, K., Poschmann, H., Bagschik, K., et al. 1999, BUSCA: a simultaneous 4 colour camera with $4 \mathrm{k} \times 4 \mathrm{k}$ CCDs, in: Optical Detectors for Astronomy. II: State-of-the-Art at the Turn of the Millenium. 4th ESO CCD Workshop, 1999, held in Garching, Germany, ed. P. Amico, \& J. W. Beletic, ISBN 0-7923-6536-4 (Dordrecht: Kluwer Academic Publishers), 143

Saffer, R. A., Bergeron, P., Koester, D., \& Liebert, J. 1994, ApJ, 432, 351

Saffer, R. A., Green, E. M., \& Bowers, T. P. 2001, The Binary Origins of Hot Subdwarfs: New Radial Velocities, in Proceedings of the Twelfth European Workshop on White Dwarf Stars, ed. H. L. Shipman, J. L. Provencal, J. MacDonald, \& S. Goodchild (San Francisco: Astronomical Society of the Pacific), ASP Conf. Ser., 226, 408

Scargle, J. D. 1982, ApJ, 263, 835

Schuh, S., Dreizler, S., Deetjen, J. L., Heber, U., \& Geckeler, R. D. 1999, Baltic Astron., 9, 1068

Silvotti, R., Solheim, J. E., Gonzalez Perez, J. M., et al. 2000, A\&A, 359, 1068

Winget, D. E., Nather, R. E., Clemens, J. C., et al. 1991, ApJ, 378, 326

Woolf, V. M., Jeffery, C. S., \& Pollacco, D. L. 2002, MNRAS, 329, 497 